\title{
Analysis of Water Cooling Mechanism by Impinging Jet for Hot Rolled Wire Rod
}

\author{
Seong-Kyu SEE, Hwan-Mun CHUNG ${ }^{11}$ and Choong-Won LEE ${ }^{21}$ \\ Technical Research Laboratories, POSCO, Pohang, Gyeongbuk,790-785, Korea. E-mail: seesk@posco.co.kr \\ 1) Graduate School, Kyungpook National University, Bukgu, Daegu, 1370, Korea. \\ 2) Department of Mechanical Engineering, Kyungpook National University, Bukgu, Daegu, 1370, Korea.
}

(Received on October 6, 2004; accepted in final form on January 14, 2005)

\begin{abstract}
The pre-cooling system used in the cooling process of the hot rolled wire rod is a system that sprays cooling water at high pressure to cool the fast-moving wire rod to a required temperature when the rod passes through the cooling nozzle of the cooling box. The influence of the flow phenomenon of this cooling nozzle affecting the rod temperature and the factors affecting the cooling efficiency were examined through experiments and numerical analysis. The confined-type cooling nozzle showed a phenomenon where secondary flow or vortex was produced on the flow depending on the existence of chambers and variations in the Reynolds number, and the production of such vortex affected the rod cooling efficiency phenomenon. The wire rod cooling efficiency can be said to be most effective at a point where the wire rod and water jet impinge due to flow increase, viz, the Reynolds number increase. It is believed that the optimal conditions featuring excellent cooling efficiency can be deduced in the future by analyzing the flow conditions and design conditions.
\end{abstract}

KEY WORDS: pre-cooling; hot rolled wire rod; cooling nozzle; cooling efficiency; water jet; confined type nozzle.

\section{Introduction}

Spray cooling method means a method of cooling by spraying the atomized liquid on the hot surface after jetting compressed water through a nozzle. In addition to this spray cooling method, the impinging liquid jet cooling system means a method where the jetted liquid forms a liquid film, boils and flows over the hot surface. Currently, the liquid jet cooling method is widely used in industrial applications requiring rapid cooling. Since the cooling method is directly related to the cooling efficiency, and the cooling technologies determine the quality of products, especially during the hot rolling and cooling process at steel plants, a great deal of research relating to this area has been carried out.

Under these systems, factors that affect heat transfer can be divided into the factor related to the cooling liquid used as cooling material and the factor of the cooling surface, the surface of the materials to be cooled. Factors on the cooling liquid include the nozzle geometry, flowrate, temperature, and the distance between the cooling surface and the nozzle. Factors on the cooling surface include the shape, size, and the roughness of the surface being cooled, and the surface heat resistant layers.

In actual rapid cooling of hot steel, the heat transfer phenomenon during the cooling process is unsteady due to high heat flux with hot water flowing on the hot surface. In other words, the boiling phenomenon that appears when the jetted water impinges due to high heat flux carries complicated forms. The pre-cooling system used in the cooling process of the hot rolled wire rod sprays cooling water at high pressure to cool the fast-moving wire rod to a required temperature when the rod passes through the cooling nozzle of the cooling box. Since the wire rod is hot, heat transfer is made by the film boiling on the wire rod surface where the cooling water and the wire rod make contact. Also, heat transfer is made to the film boiling layer due to the convection of the cooling water. Therefore, the wire cooling mechanism can be called convection-controlled film boiling.

Many scientists have attempted to check the heat flux in a lot of fields during the process of convection-controlled cooling. Cheng et al. ${ }^{1)}$ proved through experiments that the heat transfer coefficient does not have any relation with the liquid sub-cooling pattern when sub-cooling is sufficiently high. Zurdel, ${ }^{2)}$ on the other hand, conducted a non-stationary experiment to examine the heat transfer in the cooling zones of the hot-rolled wire, and expressed the heat transfer in the heated tube as follows:

$$
N u=14.75 \operatorname{Re}^{0.33}\left(d_{0} / d_{i}\right)^{1.5}
$$

This experiment was conducted in the turbulent flow region with the Reynolds number ranging from 150000 up to 415000. From the viewpoint of cooling efficiency, the vapor film formed by such boiling hinders heat transfer to the cooling water from the hot surface and for this reason, it is more advantageous to let new water penetrate through the 
cooling water layer and the vapor layer formed on the steel surface to make direct contact with the steel. To penetrate this flow layer, it is necessary to increase the impact power of water by increasing the impinging flux and to provide new cooling water by removing the jetted cooling water. The laminar flow cooling method was developed for this purpose. The laminar flow cooling method was classified into a free surface jet method and a submerged jet method. ${ }^{3,4)}$ In the former research, ${ }^{5)}$ the heat transfer effects of the impinging surface affected by the cooling water can be expressed based on the Nusselt number, which is closely related to the flow of the cooling water on the surface by the temperature gradient of the liquid on the impinging surface. In other words, it was announced that the relations between the Nusselt number and the Reynolds number follow the power-law as shown in the formula (1) above, and increases in proportion to the power of Re. However, since the flow characteristics caused by the complicated nozzle geometry of the actual cooling system become the factor that significantly affects the performance of the wire rod cooling, the turbulent flow featuring good cooling effeciency is generally used at the actual site. Complicated nozzle geometry is used (i.e. confined type or semi-confined cooling nozzle type). Even though Suresh V. et al., ${ }^{6}$ C. F. Ma carried out research into the cooling efficiency with respect to the simple nozzle geometry, there are no current studies being conducted on the various method that the flow varies according to the geometric shape of the nozzle used in the actual cooling nozzle. A free surface jet or submerged jet is formed according to the flowrate because of these nozzle characteristics. The results of this research demonstrated that with the free surface jet, the smaller the heat flux, a region where temperature rises rapidly appears due to the hydraulic jump. As a result, the reduction in the fluid velocity, which varies according to the distance from the stagnation point where the jet sprayed from the nozzle slit impinges against the cooling wall, causes boiling and decreased cooling effects.

This research used the confined jet type as the nozzle in the wire rod cooling system. Unlike plates, the behavior of the turbulent flow is complicated by the creation of a secondary flow vortex and the behavior of the particles that are broken up into droplets after impinging with the rod. These factors may affect the cooling characteristics. Hence, the purpose of this research is to study the factors that affect these cooling characteristics and variations in flow velocity.

\section{Experimental Procedure}

Figure 1(a) shows a schematic diagram of the experimental system used to observe the flow phenomenon and examine the internal velocity. The operating fluid was water at a temperature between $10^{\circ} \mathrm{C}$ and $15^{\circ} \mathrm{C}$. The fluid compressed at the $202650 \mathrm{~Pa}$ by the pump is jetted to the nozzle after passing through the main pipe and the by-pass line. To prevent temperature rise during operation, a heat exchange tube was installed inside the water reservoir to circulate cooling water. The by-pass line was divided into 2 parts to widely control the flowrate. The range of adjusting flowrate was from $0.8 \mathrm{~m}^{3} / \mathrm{h}$ to a maximum of $5 \mathrm{~m}^{3} / \mathrm{h}$.

To measure the internal velocity of the nozzle, 1-D hot

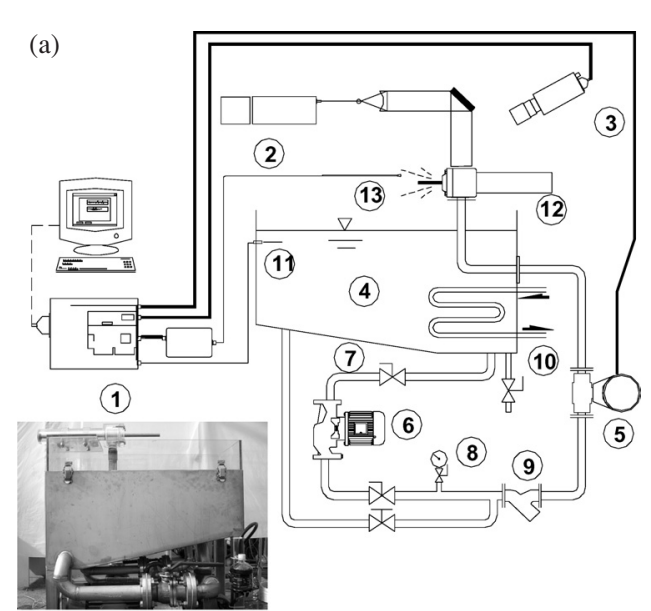
1. Data acquisition system (NI)
2. Argon-Ion Laser, 3. CCD Camera, 4. Water tank
5. Magnetic flowmeter, 6. Pump, 7. Valve
8. Pressure gauge,
9. Strainer
10. Cooling water,
11. Thermocouple
12. Nozzle,
13. Hot film

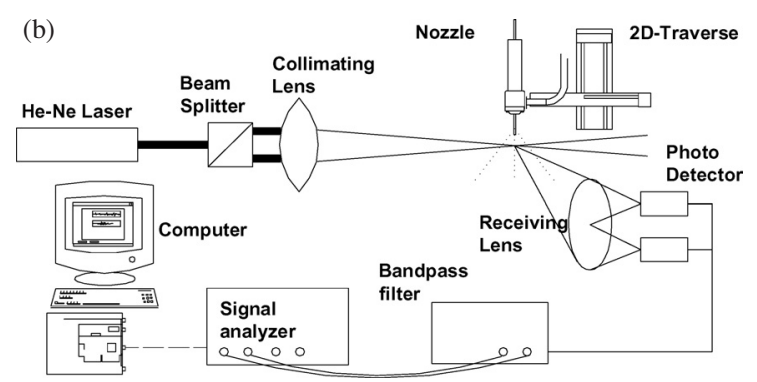

Fig. 1. Schematic diagram of (a) experimental apparatus and (b) typical LDV system.

film was used with a 3-axis traverse for the transfer of position. Since the contact angle between the actual nozzle and the wire rod is 30 degrees, the pipe was tilted at an angle of 30 degrees.

Considering the contact angle error of the hot film sensor, the sensor was inserted into the pipe to calibrate velocity. Because the frequency band does not exceed $100 \mathrm{~Hz}$ in the velocity region of this experiment, data were logged at a sampling rate of $2 \mathrm{kHz}$. The measured value of the temperature and the flowrate signal of the flow meter were simultaneously logged using the 12-bit DAQ system.

To measure the velocity at the nozzle exit, a 1-D LDV system of Aerometrics was used. Figure 1(b) shows the LDV system. The velocity measuring system used for this research is composed of a laser, beam splitter/expander, transmitter, receiver, signal analyzer, and data processor. A $15 \mathrm{~mW}$ He-Ne laser was used.

A 2-D traverse was used to move the position of the nozzle as shown in Fig. 1(a). The RTD type temperature sensor and the K-type thermocouple were used to adjust the water temperature within $\pm 1^{\circ} \mathrm{C}$ considering the temperature control range (a maximum of $50^{\circ} \mathrm{C}$ ). The magnetic flowmeter was used and the flowrate signal emitted from the output signal was averaged to determine the value. A digital cam- 


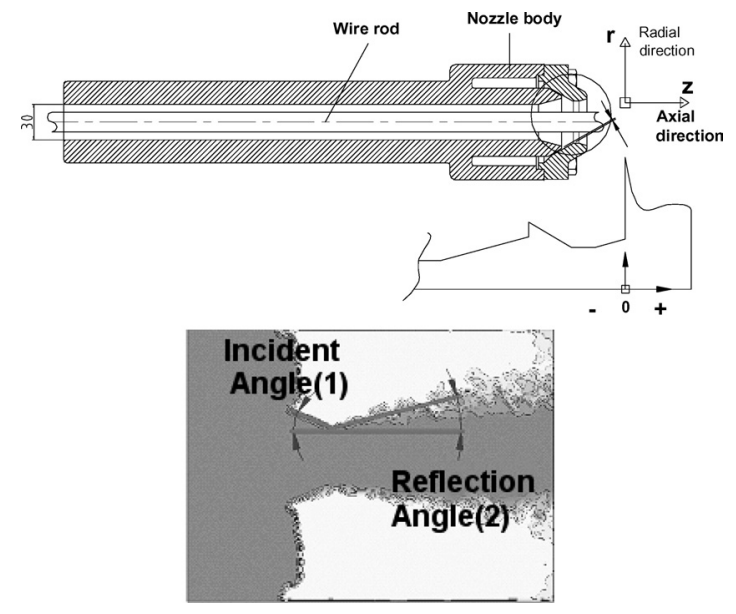

Fig. 2. Pre-cooling nozzle and calculation coordinate.

era and CCD camera were used for the image visualization system. The sheet beam of the Argon Laser was used as the light source to photograph internal flow. The numerical result was calculated using the Fluent 6.0, the commercial package, and the velocity measured value of internal flow were compared to analyzed results.

Figure 2 shows the pre-cooling nozzle used in this experiment. The slit angle is 30 degrees, with a nozzle slit width of $1.3 \mathrm{~mm}$ and a total length of $450 \mathrm{~mm}$. The nozzle is designed to allow adjustment of the variation in the nozzle clearance.

As shown in the following figure, this research defined the impinging angle as the Incident Angle and the Reflection Angle.

\section{Experimental Results and Discussion}

\subsection{External Flow}

Variation in the amount of cooling water is a factor that significantly affects the cooling efficiency. The cooling temperature is controlled by adjusting the amount of water in the actual production process.

Figure 3 shows the impinging angle varying according to the increase in flowrate. Figure 3(a) shows the variation of the impinging angle prior to impinging and 3(b) after impinging. The incident angle decreases as the flow increases prior to impinging. Conversely, the reflection angle increases as the flowrate increases after impinging. Such variation in the impinging angle of the slit jet carries trends different from those of the wall jet of the existing research featuring small flowrate, and is characterized by being dispersed into small droplets on the external part of the nozzle after impinging. In other words, if the flowrate is small based on the stagnation point, the impinging point, a laminar flow in parallel with the wire rod exists prior to and after impinging; however, in the case of actual cooling system featuring large flowrate. We can expect that the cooling performance between the flow in parallel with the wire rod inside the nozzle and the region of the droplets dispersed to the external part of the nozzle after impinging will be significantly different.

Figures 3(c) and 3(d) show the variation of the impinging angle with respect to the wire rod size, which in turn shows that the variation in the incident angle is less affected by

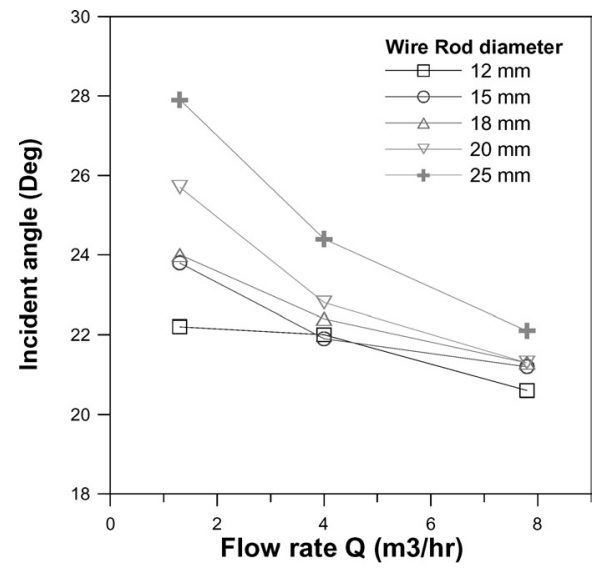

(a) Incident angle vs flowrate

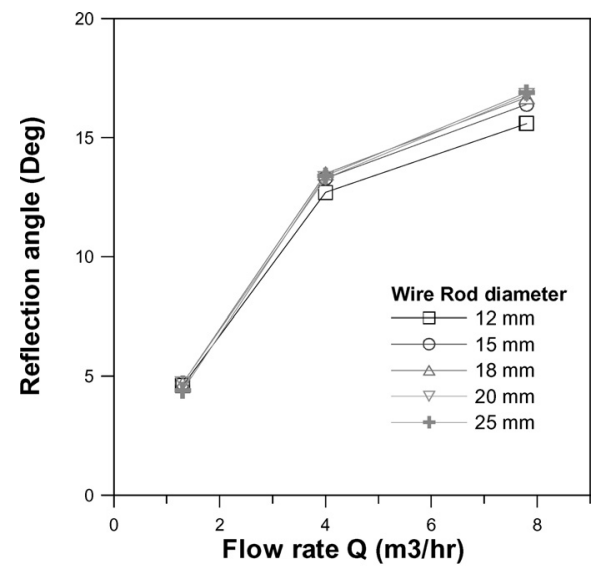

(b) Reflection angle vs flowrate

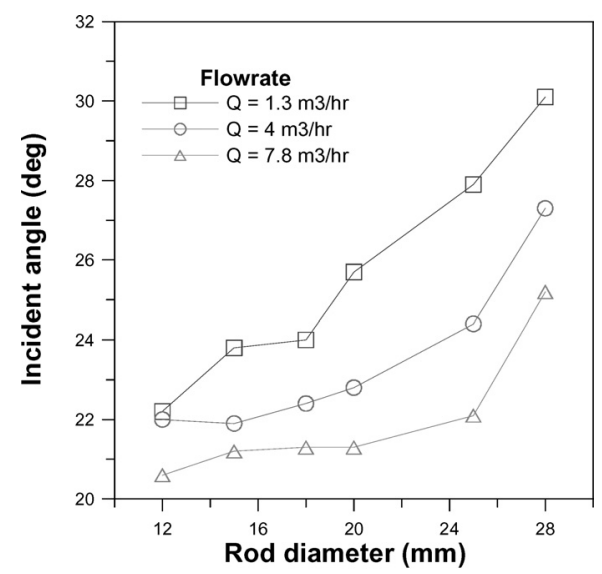

(c) Incident angle effect for variation of rod diameter

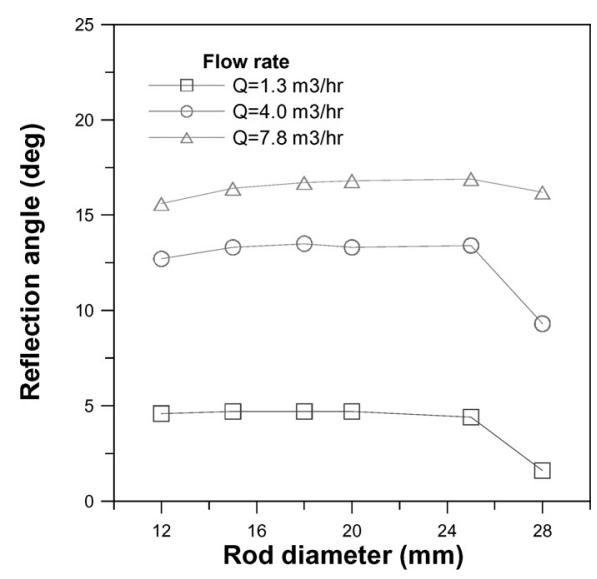

(d) Reflection angle effect for variation of rod diameter

Fig. 3. Impinging angle variation for pre-cooling nozzle. 


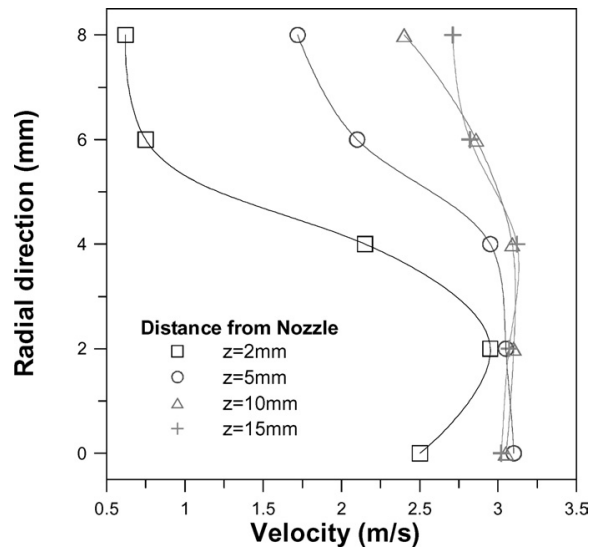

Fig. 4. LDV nozzle exit velocity measurement $\left(Q=3 \mathrm{~m}^{3} / \mathrm{h}\right)$.

smaller rod diameters, but the variation in the reflection angle remains nearly the same, regardless of the wire diameter. It is believed that such effects can be disregarded because in an actual cooling system the droplets dispersed after impinging are rarely reattached to the rod.

Figure 4 shows the result of measuring with an LDV the exit velocity distribution at the nozzle exit when the flowrate increases, and the radial direction distance from the wire rod surface varies. The same velocity pattern is maintained as the flowrate increases, and the velocity distribution becomes smooth farther from the nozzle exit and the more $z$ value increases, indicating the highest velocity at a radial direction $r=2 \mathrm{~mm}$. From the point of approximately $2 \mathrm{~mm}$, the reduction in the radial direction velocity was significant and was caused by the wall boundary effect.

Figure 5 shows the result of measuring with a LDV system the variation in the radial direction velocity when the flowrate increases at an axial distance of $2 \mathrm{~mm}$ and $10 \mathrm{~mm}$ from the nozzle. Figure 5(a), having a distance of $z=2 \mathrm{~mm}$, shows that velocity decreases after increasing when flowrate increases, while from the position of $z=10 \mathrm{~mm}$ in Fig. 5(b), velocity steadily increased linearly with only a small difference in the velocity in the radial direction compared with $2 \mathrm{~mm}$. This indicates that the velocity variation level in the radial direction was high at a point immediately in front of the nozzle exit compared with the position of $10 \mathrm{~mm}$. Because the flow direction changed according to the increase in flowrate. For this reason, the flowrate and velocity do not increase in proportion at certain flowrates.

\subsection{Internal Flow Visualization}

Figures 6(a) and 6(b) show the photograph of the flow phenomenon of the cooling system having an internal chamber. Both Figs. 6(a) and 6(b) have the form of submerged jet, and velocity is reduced due to the wall shear stress effect compared with the free surface jet, which is expected to deteriorate the cooling performance.

Figure 6(a)'s indication of slow flow also reveals the smooth flow on the wire rod without any vortex or secondary flow irrespective of whether or not there is a chamber. On the other hand, a vortex forms in the flow when the flowrate increases.

Figure 7 shows the photographs of the variation in the internal flow for the various flowrate. When a nozzle with the flowrate of $Q=2 \mathrm{~m}^{3} / \mathrm{h}$ or more was used in this experi-

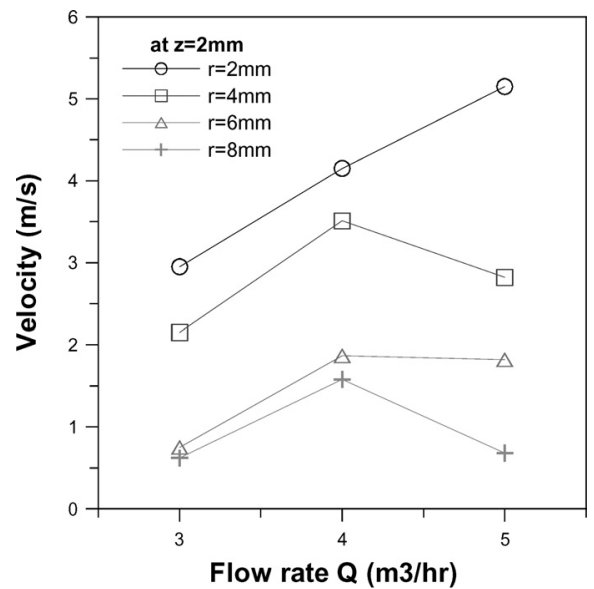

(a) Flowrate effect at $\mathrm{z}=2 \mathrm{~mm}$

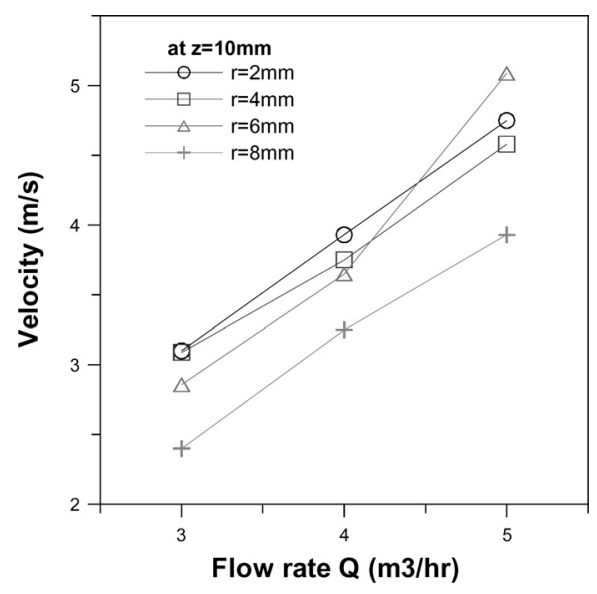

(b) Flowrate effect at $\mathrm{z}=10 \mathrm{~mm}$

Fig. 5. Flowrate effect at different position.

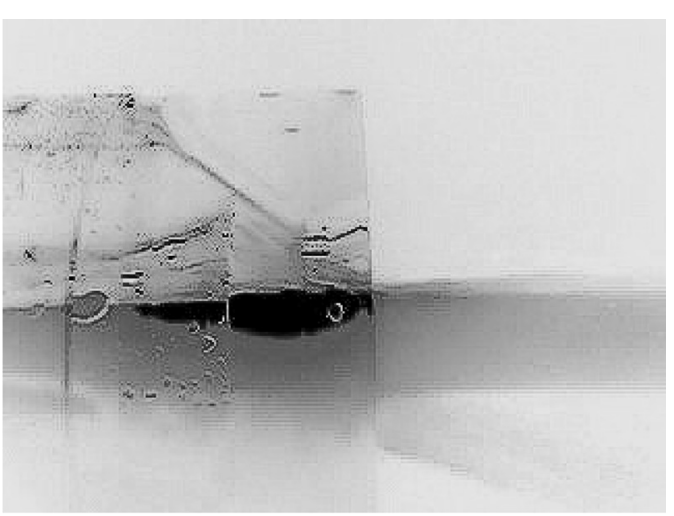

(a) $\mathrm{Q}=2 \mathrm{~m}^{3} / \mathrm{hr}$

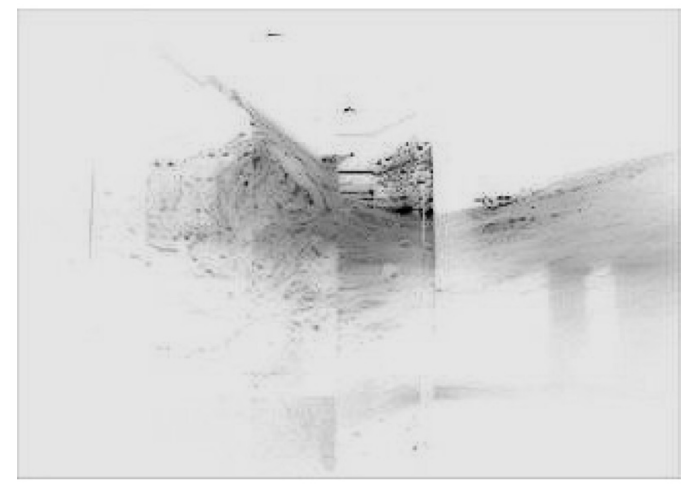

(b) $Q=4 \mathrm{~m}^{3} / \mathrm{hr}$

Fig. 6. Photographs of flow in the pre-cooling nozzle. 


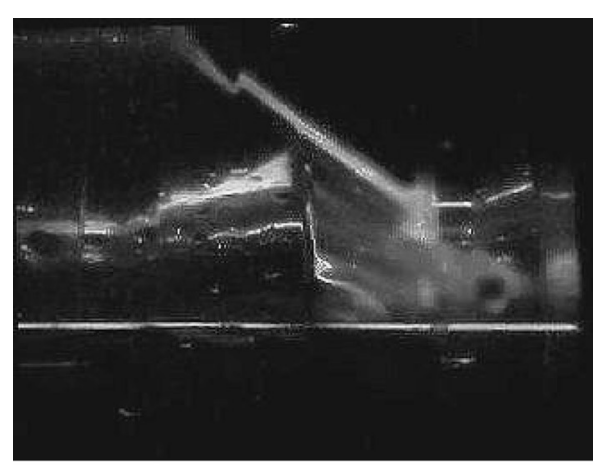

(a) $\mathrm{Q}=1.15 \mathrm{~m}^{3} / \mathrm{hr}$

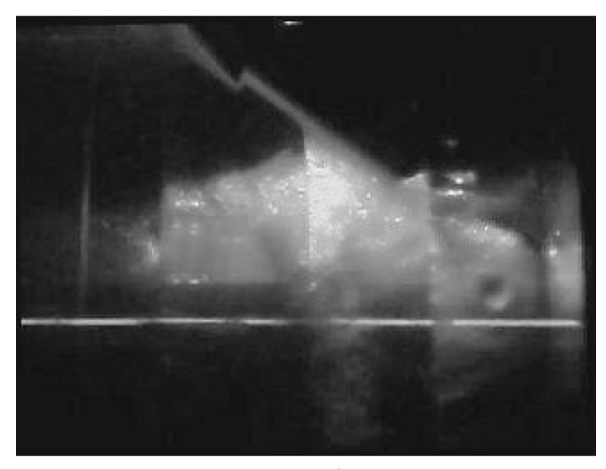

(b) $\mathrm{Q}=2.3 \mathrm{~m}^{3} / \mathrm{hr}$

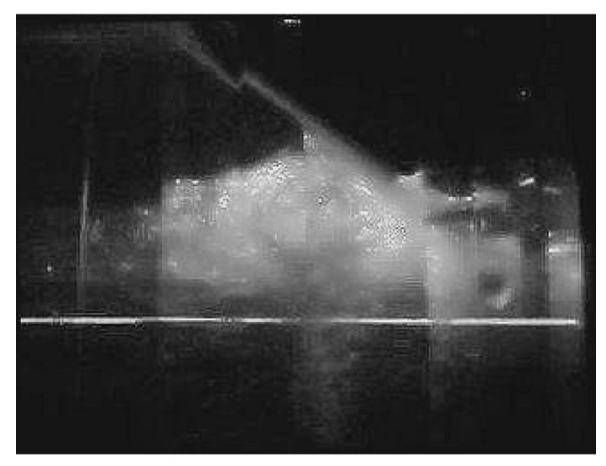

(c) $Q=3.25 \mathrm{~m}^{3} / \mathrm{hr}$

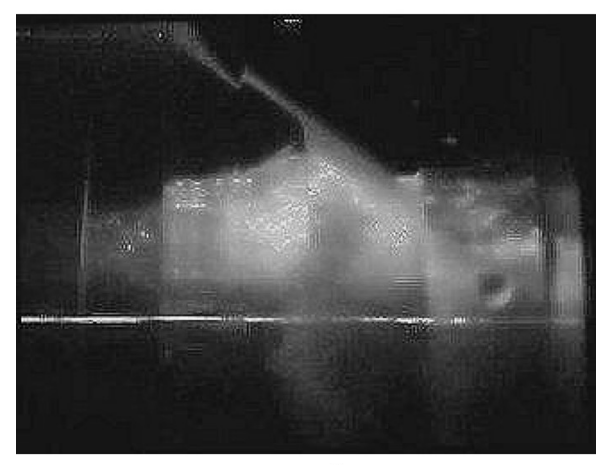

(d) $\mathrm{Q}=4.0 \mathrm{~m}^{3} / \mathrm{hr}$

Fig. 7. Photographs of internal flow for the variation of flowrate.

ment, vortexes and a secondary flow were formed. We also observed that when the flowrate increases, the internal pressure varies leading to a significant increase in cavitation.

\section{Analytical Procedure}

\subsection{Calculation Condition}

As in other numerical analyses, the basic governing equation required for the numerical analysis of the precooling system include the mass conservation equation,

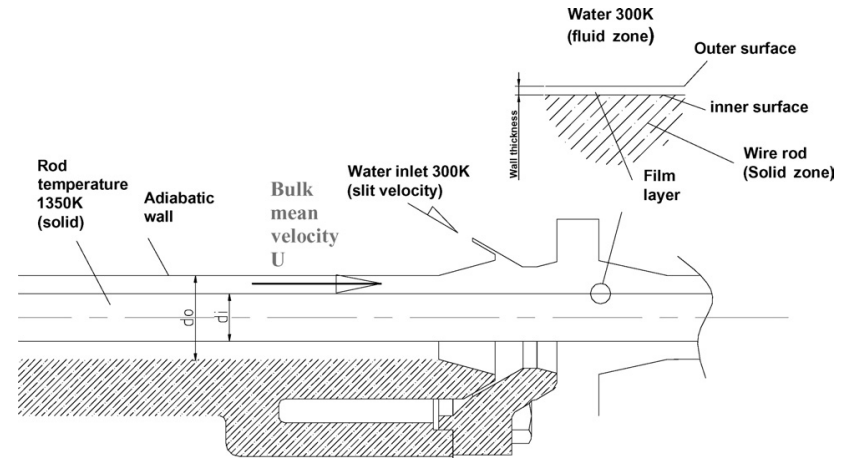

Fig. 8. Described problem in nozzle and specified thermal condition on the outside of a thin wall for coupled methods.

momentum conservation equation and the energy conservation equation relations between mass, momentum, and energy conservation. To explain the transport phenomena of the injection nozzle analyzed in this study, standard $k-\varepsilon$ Model (the turbulent model) ${ }^{8)}$ the moving reference frame model $^{9)}$ and the convection model (the heat transfer model ${ }^{10)}$ were used.

In this research, a previously developed and commercialized program (fluent 6.0) in the form of a 2-dimensional confined impinging slot jet was used to analyze the wire rod pre-cooling system. Computations have been performed over 320000 cells. A non-uniform triangular grid on the stagnation region was used, fine grids are used at the stagnation-point region and grid size increases geometrically sideway in each point. In the vertical direction, grid size increases geometrically from interface between solid and water liquid region, except for stagnation area what minimum cell area $2.723073 \mathrm{e}-07 \mathrm{~m}^{2}$.

Figure 8 depicts the boundary problem. Cooling water temperature is $300 \mathrm{~K}$. The operating conditions consisted of a pressure of $101325 \mathrm{~Pa}$ and gravity at $-9.81 \mathrm{~m} / \mathrm{s}^{2}$ in the $r$ direction. The initial temperature of steel rod is $1350 \mathrm{~K}$. Cooling water is atmospheric pressure and $300 \mathrm{~K}$. The walls of the enclosure were modeled as adiabatic walls. The interface of between the rod surface and cooling water were modeled as coupled. Coupled means that the zones on both sides of these components are coupled together and the heat transfer is calculated directly from the solution in the adjacent cells without specifying any additional boundary conditions, hence for the case of conduction problem. We can specify the material type as vapor film, wall thickness as film thickness.

The heat transfer mechanism of the pre-cooling system to be analyzed is the heat transfer based on the convection controlled film boiling as observed in the theoretical examination. The pre-cooling nozzle to be analyzed may also be classified by moving wire rod zone, the film layer zone on top of the wire rod, and the cooling water zone. Since the thickness of the wire rod zone is determined at the finishing mill, the size of the wire rod is considered fixed at the rolling prior to the finishing mill. The diameter of the wire rod was assumed to be $17 \mathrm{~mm}$ in this analysis when passing through the pre-cooling box. Because the thickness of the vapor film layer is smaller than 1/1000 of the cooling nozzle thickness, heat transfer caused by convection and radiation is ignored. Hence, the thickness of the film layer can be 


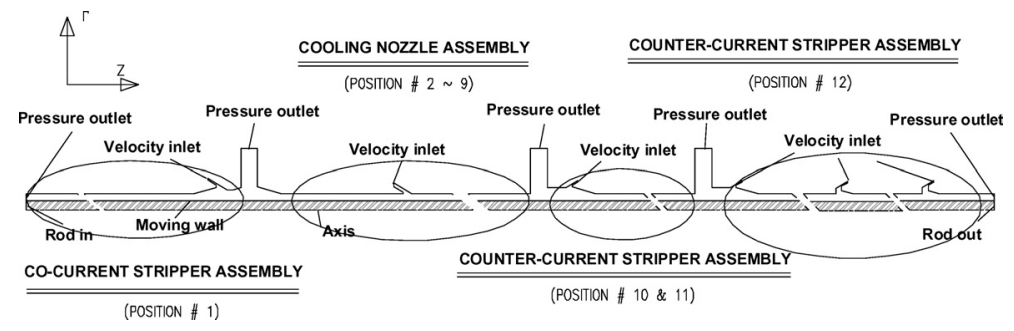

Fig. 9. Boundary condition of consecutive pre-cooling system.

expressed in average thickness considering the heat flux of the film layer. The average thickness of the vapor film can be calculated by applying Fourier's law considering the heat transfer mechanism on the surface of materials when the cooling nozzle of the wire rod is being cooled. If the actual film thickness is calculated based on this method, the result may have a value of several micrometers; however, $0.07 \mathrm{~mm}$ was decided as the basic value when the solid film layer was taken into account considering only the conductivity of the numerical analysis.

Vapor film thickness $s_{\mathrm{g}}$ averaged over length $L$ can be described approximately by following Eq. (2) ${ }^{11)}$

$$
s_{\mathrm{g}}=\frac{\lambda_{\mathrm{g}}\left(\bar{T}_{\mathrm{g}}\right)}{L} \int_{0}^{L} \frac{T_{\mathrm{w}}-T_{\mathrm{s}}}{q} d z
$$

J. Schmidt et al. ${ }^{12)}$ shows a linear dependence of the mean heat flux on Reynolds number of the liquid flow, which can be expressed by

$$
q_{\mathrm{m}}=\left(74 \times 10^{-6} \mathrm{Re}_{\mathrm{H}}-0.6\right) \quad\left(\mathrm{MW} / \mathrm{m}^{2}\right)
$$

Surface temperature of wire rod $T_{\mathrm{w}}$ is vary linearly, which can be expressed from measured temperature data by

$$
T_{\mathrm{w}}=1345-27.82 z \quad(\mathrm{~K}) .
$$

Averaged saturation temperature with pressure is $374.5 \mathrm{~K}$. Vapor film thickness can be expressed by

$$
s_{\mathrm{g}}=\frac{\lambda_{\mathrm{g}}\left(\bar{T}_{\mathrm{g}}\right)}{L} \int_{0}^{L} \frac{960.5-27.82 z}{q} d z
$$

Total length of pre-cooling zone $L$ is $5.584 \mathrm{~m}$. Vapor phase averaged temperature $\bar{T}_{\mathrm{g}}$ is averaged value of wire rod saturation temperature and cooling water. That is $840 \mathrm{~K}$. At that temperature, vapor phase thermal coductivity $\lambda_{\mathrm{g}}$ is $0.0628(\mathrm{~W} / \mathrm{m} \cdot \mathrm{K})$.

Heat flux $\dot{q}$ is obtained by J. Schmidt's experimental results. In the Eq. (3), $\operatorname{Re}=U \cdot d_{\mathrm{H}} / v$.

Bulk mean velocity $U$ is approximately $1.40 \mathrm{~m} / \mathrm{s}$, hydraulic diameter $d_{\mathrm{H}}$ is $13 \mathrm{~mm}$ and kinematic viscosity $v$ is $0.98 \times 10^{-6}\left(\mathrm{~m}^{2} / \mathrm{s}\right)$. Bulk velocity $U$ that flows in the cooling box and hydraulic diameter $d_{\mathrm{H}}$ that flows between nozzle and steel rod is used. Using Eq. (3), heat flux $\dot{q}$ is $0.77 \mathrm{MW} / \mathrm{m}^{2}$ for Reynolds number 18570 . Eventually calculated film thickness from Eq. (5) is approximately $0.07 \mathrm{~mm}$.

Figure 9 shows the calculation conditions used in the 2D analysis to analyze the heat transfer mechanism of the cooling system in the actual hot-rolled wire rod process. The

\begin{tabular}{|c|c|c|c|c|}
\hline \multicolumn{3}{|c|}{ Geometry } & \multicolumn{2}{|c|}{ xisymmetric, 2-D } \\
\hline \multicolumn{2}{|c|}{ Turbulent Model } & \multicolumn{3}{|c|}{$\mathrm{k}-\varepsilon$ model, } \\
\hline \multicolumn{5}{|c|}{ Boundary Condition } \\
\hline $\begin{array}{c}\text { Co- } \\
\text { current } \\
\text { stripper } \\
\quad \# 1 \\
\end{array}$ & $\begin{array}{c}\text { Cooling } \\
\text { nozzle } \\
\# 2-\# 9\end{array}$ & $\begin{array}{c}\text { Count- } \\
\text { current } \\
\text { stripper } \\
\# 10 \\
\end{array}$ & $\begin{array}{c}\text { Count- } \\
\text { current } \\
\text { stripper } \\
\# 11 \\
\end{array}$ & $\begin{array}{c}\text { Count- } \\
\text { current } \\
\text { stripper } \\
\# 12 \\
\end{array}$ \\
\hline $\begin{array}{c}16 \\
\mathrm{~m} / \mathrm{s}\end{array}$ & $\begin{array}{c}10 \\
\mathrm{~m} / \mathrm{s}\end{array}$ & $\begin{array}{c}16 \\
\mathrm{~m} / \mathrm{s}\end{array}$ & $\begin{array}{c}16 \\
\mathrm{~m} / \mathrm{s}\end{array}$ & $\begin{array}{c}16 \\
\mathrm{~m} / \mathrm{s}\end{array}$ \\
\hline
\end{tabular}
coordinates are $z=0$ at the rod inlet part, and $z=5.584 \mathrm{~m}$ at the rod outlet part.
Table 1. Inlet boundary condition.

Table 2. Calculation condition.

\begin{tabular}{|c|c|c|}
\hline $\begin{array}{c}\text { Wire rod } \\
\text { velocity }(\mathrm{m} / \mathrm{s})\end{array}$ & $\begin{array}{c}\text { Film } \\
\text { thickness } \\
(\mathrm{mm})\end{array}$ & $\begin{array}{c}\text { Cooling No.2 } \\
\text { Inlet velocity } \\
(\mathrm{m} / \mathrm{s})\end{array}$ \\
\hline 1.66 & 0.01 & 5 \\
\hline 3.16 & 0.04 & 6 \\
\hline 4.66 & 0.07 & 7 \\
\hline 7.66 & 0.1 & 8 \\
\hline 10.66 & 0.13 & 9 \\
\hline & 0.16 & 10 \\
\hline
\end{tabular}

There are 4 types of nozzles but the interval between nozzle assemblies was set to $15 \mathrm{~mm}$. The steel rod velocity was $10.66 \mathrm{~m} / \mathrm{s}$ from $1.66 \mathrm{~m} / \mathrm{s}$. The velocity inlet was used as the fluid inlet boundary condition, and the exit used the pressure outlet conditions. Tables $\mathbf{1}$ and $\mathbf{2}$ show the inlet boundary condition and the calculation condition used in the computation.

\subsection{Internal Flow Analysis of Fluids}

To compare with the results of experiments, the flow phenomenon was examined by ignoring the vapor film formed on the rod and the boundary of fluid. The boundary condition is identical to the stripper nozzle shown in position 1 in Fig. 9, and the coordinate position of the stripper nozzle inlet, exit, and the wire rod were set as shown in Fig. 2.

Figure 10 shows the internal flow of the nozzle when the flowrate of the cooling nozzle is $Q=2 \mathrm{~m}^{3} / \mathrm{h}$, and the initial turbulent intensity is $2.5 \%$. Figure 10 (a) indicates velocity, 10(b) the velocity vector, 10(c) pressure, and Fig. 10(d) the turbulent intensity. As shown in 10(a) and 10(b), the highest velocity is formed along the wall from the fluid inlet of the nozzle. Note that a large vortex is formed after impinging with the wire rod where the turbulent intensity reaches its maximum. In addition, negative pressure is formed, as shown in Fig. 10(c), which indicates the pressure distribution. Based on this, we can expect that cavitation may occur 


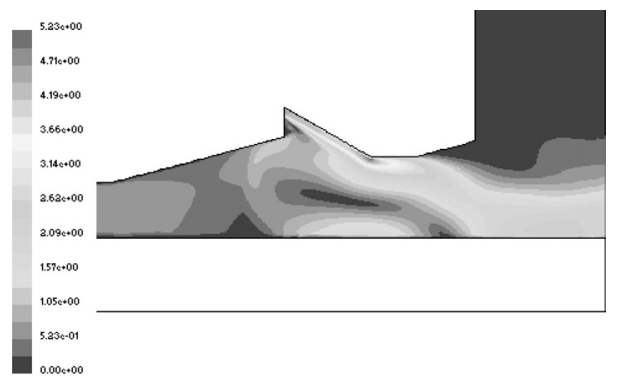

(a) Velocity contour

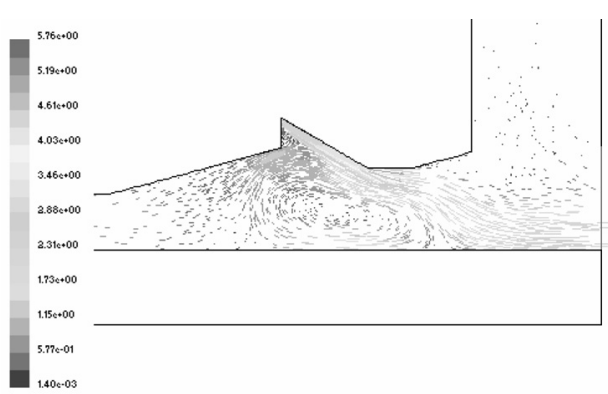

(b) Velocity vectors

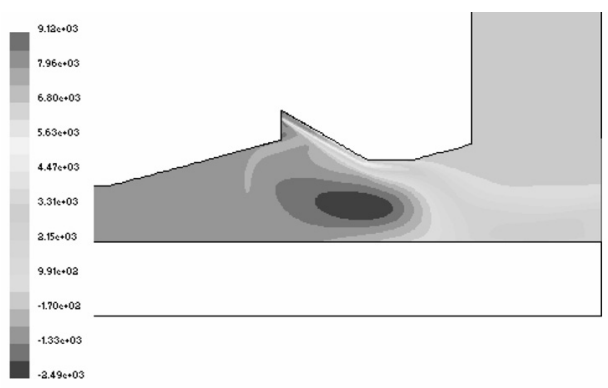

(c) Pressure contour

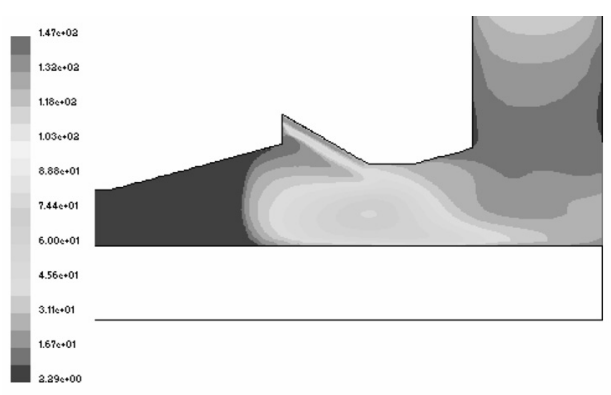

(d) Turbulent intensity (\%)

Fig. 10. Internal flow pattern of cooling nozzle.

due to the flow phenomenon.

Figure 11 shows the internal velocity and pressure distribution when the flowrate is $2 \mathrm{~m}^{3} / \mathrm{h}$ and $4 \mathrm{~m}^{3} / \mathrm{h}$. The internal velocity and pressure were indicated at an interval of $10 \mathrm{~mm}$ for the nozzle position variation from $420 \mathrm{~mm}$ $(z=-30 \mathrm{~mm})$ to $450 \mathrm{~mm}(z=0)$. From the figure, the vertical axis represents the radius variation of the nozzle, and the horizontal axis, the velocity and the amount of pressure. As shown by the radial pressure distribution, negative pressure may react by the formation of a vortex on the nozzle. In addition, from the nozzle outlet, a point $0 \mathrm{~mm}$ from the $z$-axis, velocity increases starting from the nozzle surface to a point approximately $4 \mathrm{~mm}$ in the radial direction, and the highest velocity maintains a value of approximately $2 \mathrm{~m} / \mathrm{s}$ when the flowrate is $Q=2 \mathrm{~m}^{3} / \mathrm{h}$, and approximately $4 \mathrm{~m} / \mathrm{s}$

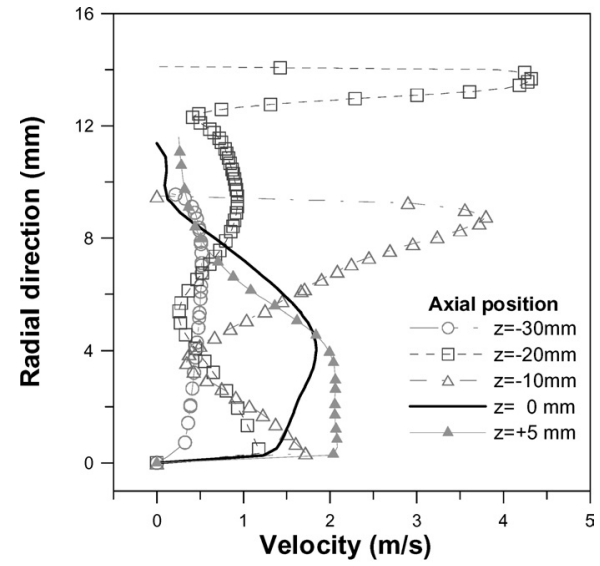

(a) Velocity magnitude for $\mathrm{Q}=2 \mathrm{~m}^{3} / \mathrm{hr}$

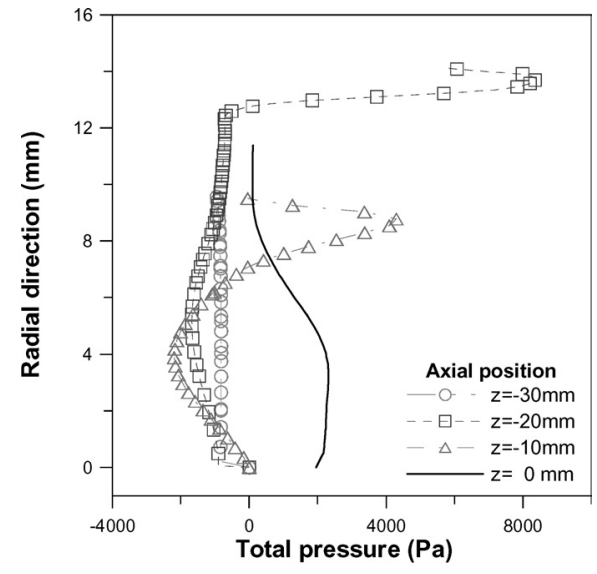

(b) Total pressure for $\mathrm{Q}=2 \mathrm{~m}^{3} / \mathrm{hr}$

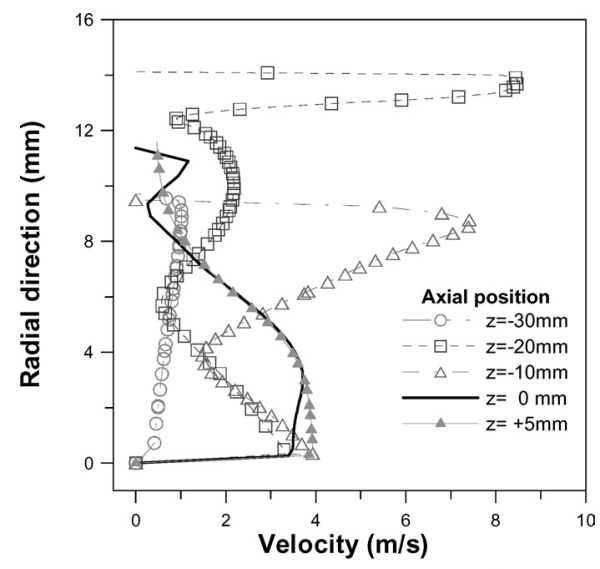

(c) Velocity magnitude for $\mathrm{Q}=4 \mathrm{~m}^{3} / \mathrm{hr}$

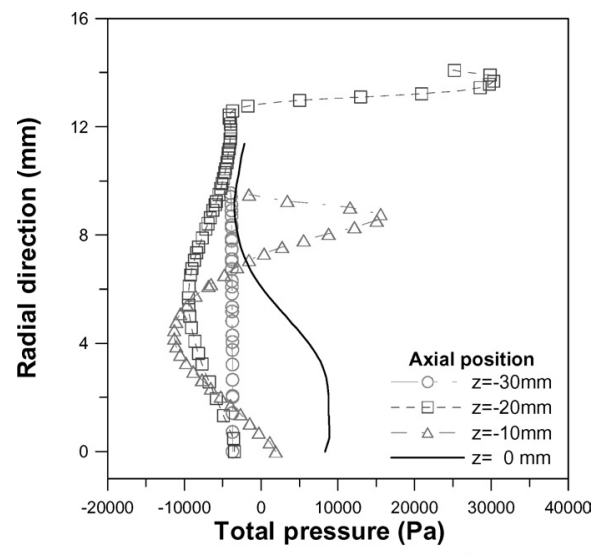

(d) Total pressure for $\mathrm{Q}=4 \mathrm{~m}^{3} / \mathrm{hr}$

Fig. 11. Velocity and total pressure magnitude in co-current stripper nozzle for $Q=2 \mathrm{~m}^{3} / \mathrm{h}$ and $4 \mathrm{~m}^{3} / \mathrm{h}$. 
when the flowrate is $Q=4 \mathrm{~m}^{3} / \mathrm{h}$. Then the value decreases again. At a point of $+5 \mathrm{~mm}$, indicating the external region, the velocity distribution does not show any variation up to a point $4 \mathrm{~mm}$ in the radial direction and then starts to decrease. These trends appear similar to those of the common spray nozzle. At the position of $z=-30 \mathrm{~mm}$, the velocity is low with almost no influence by the nozzle slit. At the point of $z=-20 \mathrm{~mm}$ where the slit jet is located, the velocity is $4 \mathrm{~m} / \mathrm{s}$ and $8 \mathrm{~m} / \mathrm{s}$ at a point $2 \mathrm{~mm}$ in the radial direction, indicating a value 2 times the external velocity of the nozzle after an approximate outlet $(z=0 \mathrm{~mm})$.

\subsection{Temperature Analysis}

Figures 12 to 14 show the results of analyzing the temperature under conditions specified in Tables 1 and 2 to examine factors that affect the wire rod cooling of the actual cooling system.

Figure 12 shows the rod surface temperature variation occurring when the flowrate of the cooling nozzle No. 2 through 9 is increased. In the figure, the vertical dotted line indicates the position of each nozzle. It can be understood that as the inlet velocity increases, the cooling effects increase due to the effects of the turbulent intensity increase and the vapor film layers being thinner. In addition, the difference gradually increases when moving nearer the rod outlet because the flowrate is accumulated.

Figure 13 shows the results of calculating the rod temperature when the velocity of the rod being inserted into the cooling box is changed when the vapor film thickness is $0.07 \mathrm{~mm}$. From this, we can understand that the slower the rod velocity, the greater the cooling effects. It is believed that this phenomenon occurs because the cooling efficiency has increased due to the effects of the given sufficient cooling time.

Figure 14 shows the surface temperature distribution of the rod obtained by changing the vapor film thickness. This result have indicated that the rod surface temperature increases as the film thickness increases when the wire rod velocity is $7.66 \mathrm{~m} / \mathrm{s}$. In other words, the important factor in increasing the cooling efficiency is to reduce the thickness of the water vapor.

\section{Comparison of the Experimental and Calculated Values}

Figure 15 shows a comparison between the data obtained by measuring the velocity variation with the hot film in each position when the flowrate increases, and the calculation results obtained through numerical analysis.

The variation of flowrate continued for a specified duration and the hot film data were aquired. The results of the numerical analysis and experimental results match so well. The reason that velocity variation caused by the change in the flowrate differs depending on its position is believed to have resulted from the jet sprayed from the slit and the variation of the wire rod impinging point.

Figure 16 shows the results of comparing the calculated value of the velocity distribution at the nozzle exit when the flowrate increases at $z=2 \mathrm{~mm}$ position with the LDV measurement value. The figure shows the exit velocity when the radial distance from the wire rod surface changes, indicat-

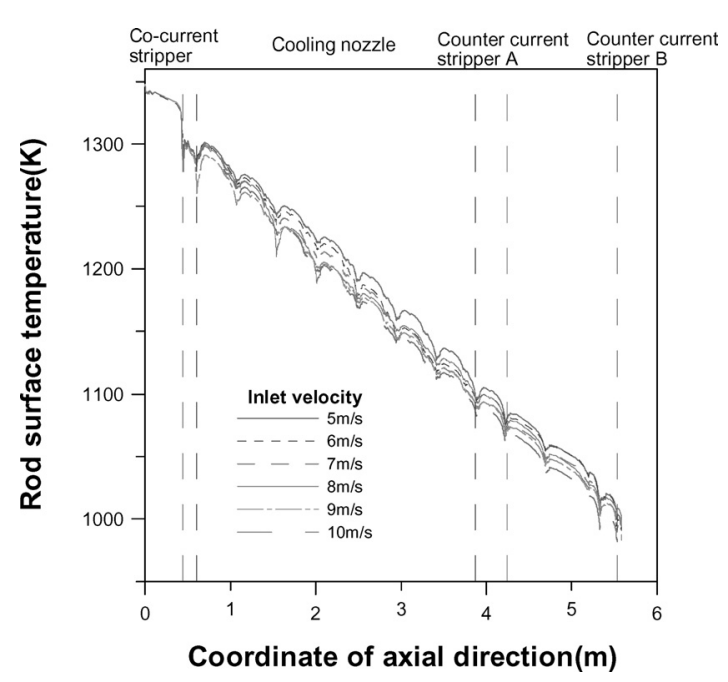

Fig. 12. Rod surface temperature for the variations of flowrate.

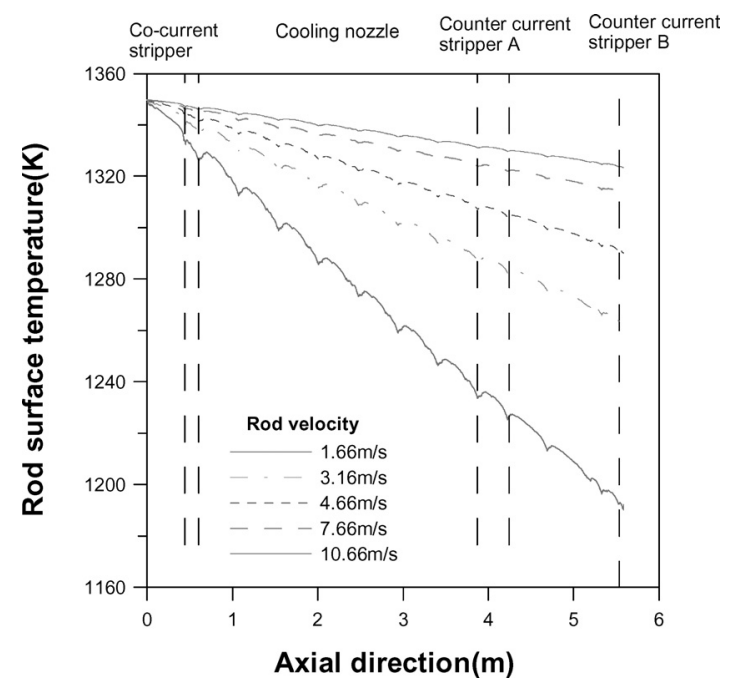

Fig. 13. Rod surface temperature $v s$. rod velocity for film thickness of $0.07 \mathrm{~mm}$.

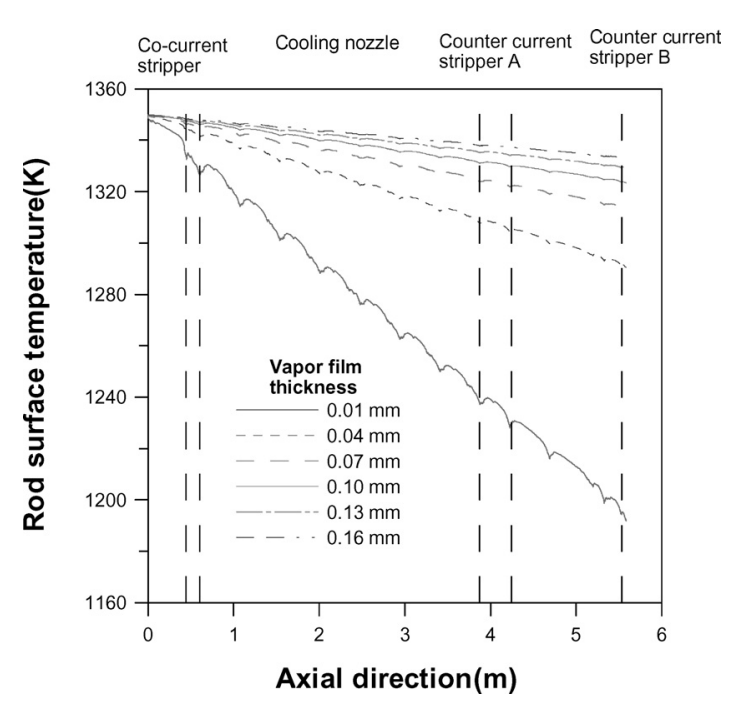

Fig. 14. Rod surface temperature for variations in vapor film thickness.

ing that both values match.

Figure 17 shows the comparison between the results of numerically analyzing the internal flow of the fluid and the 


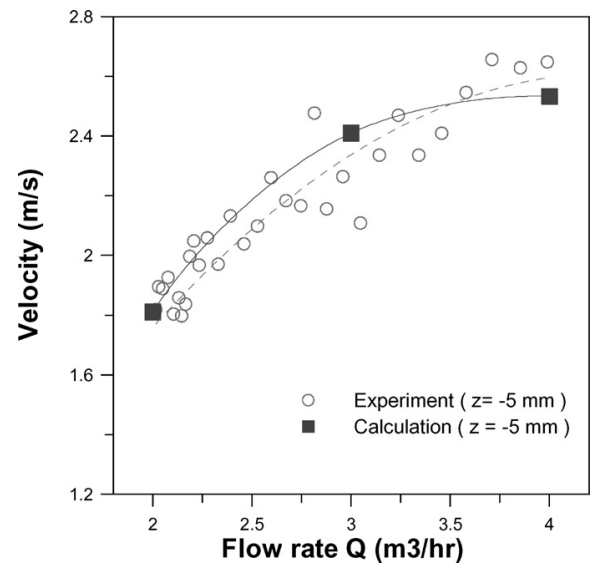

Fig. 15. Comparison of hot film velocity signal data for variations in flowrate at $z=-5 \mathrm{~mm}$.

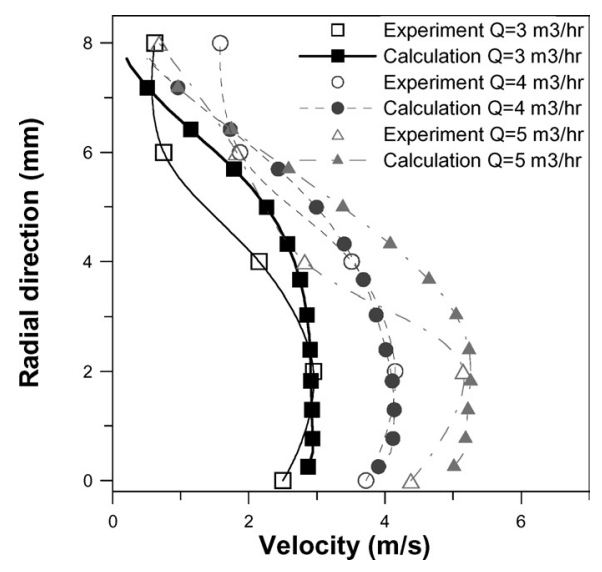

Fig. 16. Comparison for exit velocity calculation to measurement value by LDV.

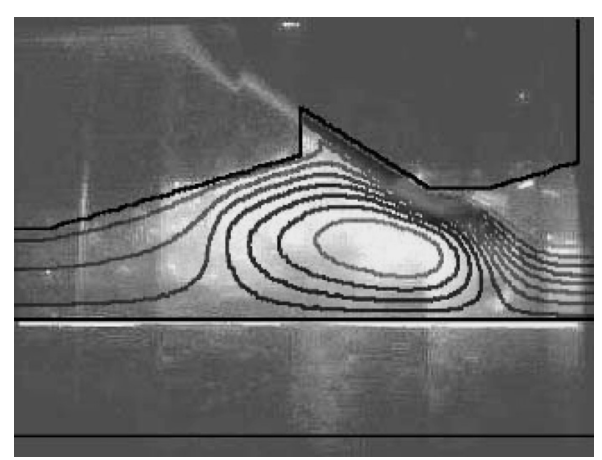

Fig. 17. Comparison of calculated vector to internal flow pattern of cooling nozzle.

experimental results based on flow visualization. When the internal flow of the nozzle and the flow photographs were compared when the flowrate of the cooling nozzle is $Q=2 \mathrm{~m}^{3} / \mathrm{h}$ and the initial turbulent intensity is $2.5 \%$, a large vortex is formed on the same part after impinging with the wire rod, indicating that the experimental results match the numerical analysis. To examine the nozzle installed on the actual pre-cooling system, the nozzle and the box actually used were manufactured at a scale of $1: 1$, and the basic tests were conducted on flow and cooling to compare the results with those obtained by numerical analysis. The heating temperature of the electric furnace in pre-cooling simulator and the heating time were set to the $1100^{\circ} \mathrm{C}$ and $1.5 \mathrm{~h}$

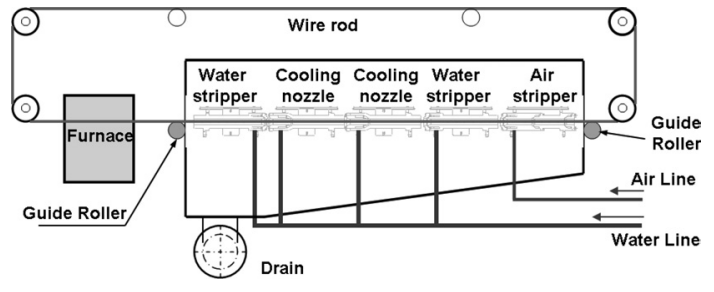

Fig. 18. Schematic diagram of cooling experimental apparatus.

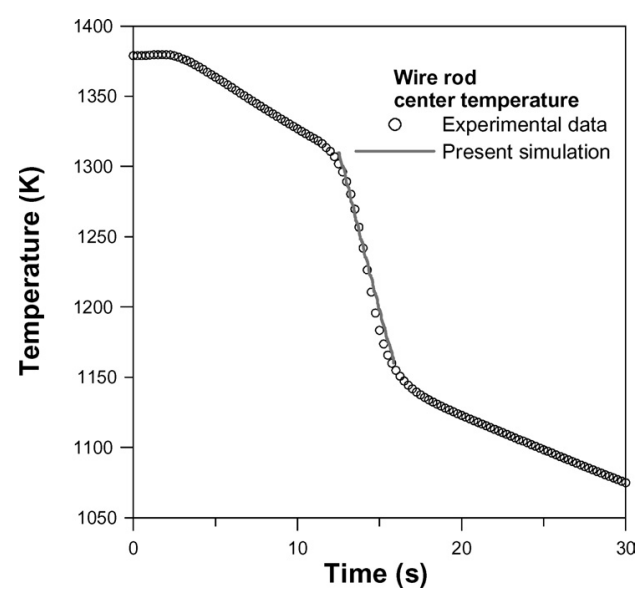

Fig. 19. Cooling curve for $17 \mathrm{~mm}$ wire rod.

to perform cooling tests. The nozzles used for the test were $450 \mathrm{~mm}$ long, and a water pump was also installed to supply the cooling water for the experiment.

Test samples were $10 \mathrm{~mm}$ and $17 \mathrm{~mm}$ stainless wire rods. A $1.6 \mathrm{~mm}$ thermocouple was inserted lengthwise and after heating the samples in the furnace, the temperature was measured when the samples were moving from the precooling simulator. Figure 18 shows the schematic diagram of the cooling experimental apparatus used in this experiment.

The results of the cooling test carried out by the experimental apparatus are indicated in the cooling curve shown in Fig. 19. The continuous line indicates the results of analyzing the rod center temperature when the vapor film thickness was $0.07 \mathrm{~mm}$ and the wire rod velocity was $1.66 \mathrm{~m} / \mathrm{s}$. The initial temperature of the wire rod inserted into the nozzle was $1310 \mathrm{~K}$ and the temperature variation according to time change was obtained by converting the temperature into the velocity of the wire rod moving $5584 \mathrm{~mm}$, the entire length of the actual cooling system. The cooling rate of approximately $104^{\circ} \mathrm{C} / \mathrm{s}$ was obtained with the $17 \mathrm{~mm}$ wire rod.

\section{Microstructure}

Cold heading quality wire is used for the manufacture of bolts, nuts, and screws. In the past, these clamps were manufactured through cutting or hot working; however, cold working is now in vogue to improve productivity and reduce costs. Therefore, the cold heading quality wire requires low tensile strength to obtain good workability and surface quality so that cold working can be performed.

This research, in respect to SCM435 materials - the structural alloy steel, compared the mechanical properties and the microstructure of commercial products and the cold 


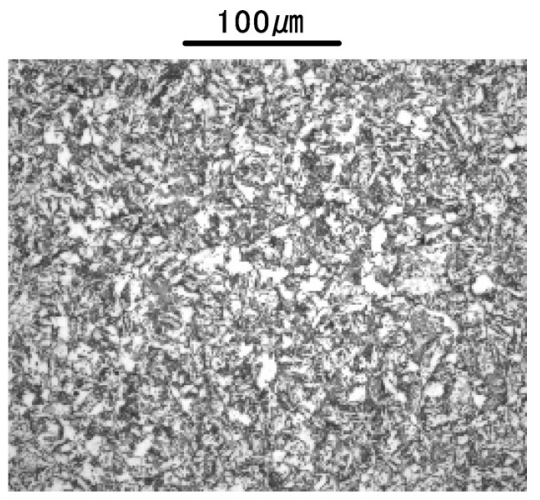

(a) Commercial products

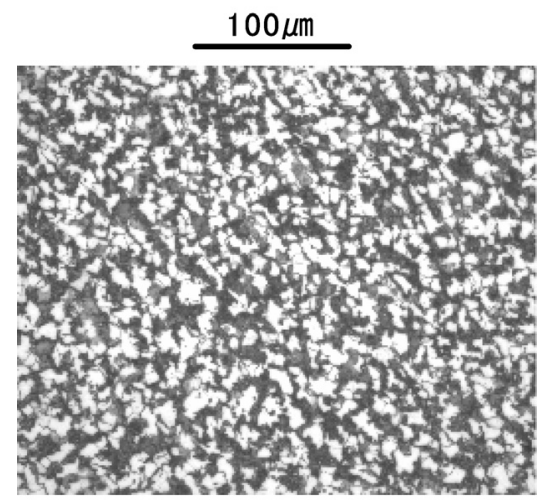

(b) Control-rolled product

Fig. 20. Comparison between the microstructure of commercial products and that of the control-rolled products.

rolled products manufactured through controlled rolling. The tensile strength (TS) of the common product, the mixture of Martensite and Bainite, was approximately 9-11 $\mathrm{MPa}$; however, in the control-rolled product, the mixture of Ferrite and Pearlite was approximately $6.8-7.3 \mathrm{MPa}$. In addition, the spheroidized annealing rate was $72 \%$ for the commercial products, while that of the control-rolled products was as high as $75 \%$.

Figure 20 shows the SEM photos comparing the microstructure of the steel surface between commercial and control-rolled products.

\section{Conclusion}

The pre-cooling system used in the cooling process of the hot rolled wire rod utilizes a spray of cooling water at high pressure to cool the fast-moving wire rod to a required temperature when the rod passes through the cooling nozzle of the cooling box. The influence of the flow phenomenon of this cooling nozzle affecting the rod temperature and the factors affecting the cooling efficiency were examined through experiments and numerical analyses. The results are as follows:

(1) The confined-type cooling nozzle demonstrated a phenomenon where secondary flow or vortex was produced on the flow depending on the existence of chambers and variations in the Reynolds number. The production of such vortex affected the rod cooling efficiency.

(2) The wire rod cooling efficiency was found to be most effective at a point where the water jet impinge the wire rod due to flow increase, that is, the Reynolds number increase

(3) The estimated results based on numerical analysis showed that the cooling efficiency was reduced as the vapor film thickness and the rod velocity increased. In other words, the rod surface temperature increases when the film thickness increases. The important factor of increasing the cooling efficiency, therefore, is to reduce the thickness of the vapor film.

(4) As a result of comparing the mechanical properties and the microstructure of the commercial and cold rolled products obtained through controlled rolling, with respect to SCM435 material - the structural alloy steel, a superior structure featuring low tensile strength and a mixture of Ferrite and Pearlite was obtained.

(5) It is believed that the optimal conditions for excellent cooling efficiency can be deduced in the future by analyzing flow and design conditions.

\section{Nomenclature}

$d$ : Nozzle slit diameter

$h$ : Heat transfer coeffecient

$N u$ : Nusselt number $\left(=h d_{\mathrm{H}} / \lambda\right)$

$q$ : Heat flux

$Q:$ Flow rate

$\operatorname{Re}_{\mathrm{H}}$ : Reynolds number of liquid flow $\left(=U d_{\mathrm{H}} / v\right)$

$r$ : Coordinate of radial direction

$s:$ Film thickness

$T$ : Temperature

$U:$ Bulk velocity

$z$ : Coordinate of axial direction

$\lambda$ : Thermal conductivity

$v$ : Kinematic viscosity

Subscript
g: Gas phase
i: Slit inner diameter
o: Slit outer diameter
$\mathrm{s}$ : Saturation
$H$ : Hydraulic diameter
w: Wire rod

\section{REFERENCE}

1) S. C. Cheng, W. W. L, Ng and K. T. Heng: Int. J. Heat Mass Transfer, 21 (1978), 1385.

2) K. Zurdel: PhD. Thesis, Technische Hochschule Magdeburg, (1974).

3) N. Hatta and H. Osakabe: ISIJ Int., 29 (1989), No. 11, 919.

4) R. Ishida, A. Mizuta, K. Korida, S. Yasunaga and K. Takisawa: ISIJ Int., 29 (1989), No. 4, 339.

5) C. Himadri and K. S. Sujoy: Int. J. Heat Fluid Flow, 24 (2003), 685.

6) V. Suresh and B. Neraydykh: Int. J. Heat Mass Transfer, 39 (1996), No. 14, 2915.

7) C. F. Ma, Q. Zheng, H. Sun and K. Wu: Int. J. Heat Mass Transfer, 40 (1997), No. 10, 2249.

8) B. E. Launder and D. B. Spalding: Comp. Methods Appl. Mech. Eng., 3 (1974), 269.

9) Fluent 6.0 User's Guide Volume 2, Fluent Inc., Lebanon, NH, (2001), 9-1.

10) Fluent 6.0 User's Guide Volume 2, Fluent Inc., Lebanon, NH, (2001), 11-1.

11) J. Schmidt: Heat Mass Transfer, 36 (2000), 265.

12) J. Schmidt: Heat Mass Transfer, 36 (2000), 165. 